\title{
Major Indices and Perfect Bases for Complex Reflection Groups
}

\author{
Robert Shwartz* \\ Department of Mathematics \\ Bar-Ilan University \\ Ramat-Gan 52900, Israel \\ shwartr1@math.biu.ac.il
}

\author{
Ron M. Adin \\ Department of Mathematics \\ Bar-Ilan University \\ Ramat-Gan 52900, Israel \\ radin@math.biu.ac.il
}

\author{
Yuval Roichman \\ Department of Mathematics \\ Bar-Ilan University \\ Ramat-Gan 52900, Israel \\ yuvalr@math.biu.ac.il
}

Submitted: Aug 16, 2007; Accepted: Apr 8, 2008; Published: Apr 18, 2008 Mathematics Subject Classification: Primary 05E15, 20F55;

Secondary 20F05, 13A50.

\begin{abstract}
It is shown that, under mild conditions, a complex reflection group $G(r, p, n)$ may be decomposed into a set-wise direct product of cyclic subgroups. This property is then used to extend the notion of major index and a corresponding Hilbert series identity to these and other closely related groups.
\end{abstract}

\section{Introduction}

\subsection{The Major Index}

Let $S_{n}$ be the symmetric group on $n$ letters. $S_{n}$ is a Coxeter group with respect to the Coxeter generating set $S=\left\{s_{i} \mid 1 \leq i<n\right\}$, where $s_{i}:=(i, i+1)(1 \leq i<n)$ are the adjacent transpositions. Let $\ell(\pi)$ be the length of $\pi \in S_{n}$ with respect to $S$, let

$$
\operatorname{Des}(\pi):=\left\{1 \leq i<n \mid \ell\left(\pi s_{i}\right)<\ell(\pi)\right\}
$$

\footnotetext{
${ }^{*}$ Research of all authors was supported in part by the Israel Science Foundation, grant no. 947/04.
} 
be the descent set of $\pi$ (where permutations are multiplied from right to left), and let

$$
\operatorname{maj}(\pi):=\sum_{i \in \operatorname{Des}(\pi)} i
$$

be the major index of $\pi$. It is well known that

$$
\ell(\pi)=\#\{i<j \mid \pi(i)>\pi(j)\},
$$

the number of inversions in $\pi$, and that

$$
\operatorname{Des}(\pi)=\{1 \leq i \leq n-1 \mid \pi(i)>\pi(i+1)\} .
$$

The major index is involved in many classical identities on the symmetric group; see, for example, $[15,11,12,8]$. The search for an extended major index and corresponding identities on other groups, initiated by Foata in the early nineties, turned out to be successful for the classical Weyl groups and some wreath products. In particular, the Hilbert series of the coinvariant algebra of the symmetric group $S_{n}$ and of the wreath products $\mathbf{Z}_{r} \prec S_{n}$ may be expressed as generating functions for the flag major index on these groups $[3,5,1]$. A generalization of this result to complex reflection groups, involving the notion of basis for a group, is suggested in this paper. This generalization extends previous results of [3].

\section{$1.2 \quad$ Bases}

The concept of basis for a group $[18,16]$ extends the classical Fundamental Theorem for Finitely Generated Abelian Groups to the non-abelian case.

Definition 1.1. Let $G$ be a finite group. A sequence $\mathbf{a}=\left(a_{1}, \ldots, a_{n}\right)$ of elements of $G$ is called $a$ basis (or a starred ordered generating system, OGS*) for $G$ if there exist positive integers $m_{1}, \ldots, m_{n}$ such that every element $g \in G$ has a unique presentation in the form

$$
g=a_{1}^{k_{1}} a_{2}^{k_{2}} \cdots a_{n}^{k_{n}}
$$

with $0 \leq k_{i}<m_{i}$ for every $1 \leq i \leq n$.

If $m_{i}=o\left(a_{i}\right)$ (the order of the element $a_{i}$ ) for every $1 \leq i \leq n$ then $\mathbf{a}$ is a perfect basis (or an ordered generating system, OGS) for $G$.

A finite group $G$ has a perfect basis if and only if $G$ has a decomposition into a set-wise direct product of cyclic subgroups. Namely, a group $G$ has a perfect basis if and only if there exist subgroups $C_{1}, \ldots, C_{n}$ of $G$ such that

$$
C_{i} \text { is cyclic } \quad(\forall i),
$$

$$
\begin{array}{r}
G=C_{1} \cdots C_{n} \text {, and } \\
C_{i} \cap\left(C_{1} \cdots \hat{C}_{i} \cdots C_{n}\right)=\{1\}
\end{array}
$$




\section{Examples:}

1. $p q$-groups ( $p, q$ distinct primes) have a perfect basis [18].

2. The group of quaternions $Q_{8}$ has a basis, but not a perfect basis [18].

The major index of a permutation has an algebraic interpretation in terms of a perfect basis. The following observation is a reformulation of [3, Claim 2.1].

Observation 1.2. Let $s_{i}:=(i, i+1) \in S_{n}(1 \leq i<n)$ and

$$
t_{i}:=s_{i} s_{i-1} \cdots s_{1} \quad(1 \leq i<n) .
$$

Then $\left(t_{n-1}, t_{n-2}, \ldots, t_{1}\right)$ is a perfect basis for $S_{n}$; namely, every permutation $\pi \in S_{n}$ has a unique presentation

$$
\pi=t_{n-1}^{k_{n-1}} \cdots t_{1}^{k_{1}},
$$

where $0 \leq k_{i}<o\left(t_{i}\right)=i+1(1 \leq i<n)$. In this notation,

$$
\operatorname{maj}(\pi)=\sum_{i=1}^{n-1} k_{i}
$$

This observation was applied in [3] to solve a problem of Foata regarding the hyperoctahedral group. In this paper, this approach is extended to complex reflection groups.

\section{Concepts and Results}

\subsection{Background: Wreath Products}

The colored permutation group $G(r, n)$ is the wreath product of the cyclic group $\mathbf{Z}_{r}$ by the symmetric group $S_{n}$. Namely,

$$
G(r, n)=\mathbf{Z}_{r} 2 S_{n}:=\left\{\left(\left(c_{1}, \ldots, c_{n}\right) ; \pi\right) \mid c_{i} \in \mathbf{Z}_{r}, \pi \in S_{n}\right\}
$$

with group operation

$$
\left(\left(c_{1}, \ldots, c_{n}\right) ; \pi\right) \cdot\left(\left(c_{1}^{\prime}, \ldots, c_{n}^{\prime}\right) ; \pi^{\prime}\right):=\left(\left(c_{1}+c_{\pi^{-1}(1)}^{\prime}, \ldots, c_{n}+c_{\pi^{-1}(n)}^{\prime}\right) ; \pi \pi^{\prime}\right) .
$$

Proposition 2.1. Let $\tau_{i}:=\left((1,0, \ldots, 0) ; t_{i}\right)(0 \leq i<n)$, where $t_{i}:=s_{i} \cdots s_{1} \in S_{n}$ $(1 \leq i<n)$, as in Observation 1.2 above, and $t_{0}=I d \in S_{n}$, the identity permutation. Then $\left(\tau_{n-1}, \ldots, \tau_{0}\right)$ is a perfect basis for $G(r, n)$, i.e., every element $\pi \in G(r, n)$ has a unique presentation

$$
\pi=\tau_{n-1}^{k_{n-1}} \cdots \tau_{1}^{k_{1}} \tau_{0}^{k_{0}}
$$

where $0 \leq k_{i}<o\left(\tau_{i}\right)=r(i+1)(0 \leq i<n)$. 
Proposition 2.1 generalizes the first part of Observation 1.2, which concerns the special case $G(1, n)=S_{n}$. It is a slightly modified version of a result described in [3], where the basis elements are $\tau_{0}^{-1} \tau_{i} \tau_{0}$ instead of our $\tau_{i}$.

Given the unique presentation (1), define the flag major index of a colored permutation $\pi \in G(r, n)$ by

$$
\operatorname{fmaj}_{G(r, n)}(\pi):=\sum_{i=0}^{n-1} k_{i}
$$

the sum of exponents in $(1)$.

\subsection{General Concepts}

Given a (perfect) basis $\mathbf{a}=\left(a_{1}, \ldots, a_{n}\right)$ for a group $G$, define the $(G, \mathbf{a})$ flag major index as follows. For every $g \in G$ let

$$
\operatorname{fmaj}_{(G, \mathbf{a})}(g):=\sum_{i=1}^{n} k_{i},
$$

where $k_{i}(1 \leq i \leq n)$ are the exponents in the unique presentation

$$
g=a_{1}^{k_{1}} \cdots a_{n}^{k_{n}} \quad\left(0 \leq k_{i}<m_{i}\right) .
$$

Let

$$
\operatorname{Fmaj}_{(G, \mathbf{a})}(q):=\sum_{g \in G} q^{\operatorname{fmaj}_{(G, \mathbf{a})}(g)}
$$

be the corresponding generating function.

By definition,

$$
\operatorname{Fmaj}_{(G, \mathbf{a})}(q)=\prod_{i=1}^{n}\left[m_{i}\right]_{q},
$$

where

$$
\left[m_{i}\right]_{q}:=\frac{q^{m_{i}}-1}{q-1}
$$

Given a group $G$ with a set of generators $S$, let $\ell_{(G, S)}(\cdot)$ denote the length function on $G$ with respect to $S$, that is,

$$
\ell_{(G, S)}(g):=\min \left\{\ell: g=s_{1} s_{2} \cdots s_{\ell} \text { for some } s_{i} \in S\right\} ;
$$

and let the Poincaré series of $G$ (with respect to $S$ ) be the corresponding generating function

$$
\operatorname{Poin}_{(G, S)}(q):=\sum_{g \in G} q^{\ell_{(G, S)}(g)} .
$$

The case where $(G, S)$ is a Coxeter system has been extensively studied (see, e.g., [14]). If $G$ is a Coxeter group we will always assume that $S$ is the Coxeter generating set.

Motivated by Observation 1.2 we define a (perfect) Mahonian basis for $G$ as follows. 
Definition 2.2. Let a be a (perfect) basis for a group $G$ and let $S$ be a generating set of $G$. Then a is a (perfect) Mahonian basis for $G$ with respect to $S$ if

$$
\operatorname{Fmaj}_{(G, \mathbf{a})}(q)=\operatorname{Poin}_{(G, S)}(q) ;
$$

namely, if the $(G, \mathbf{a})$ flag major index is equidistributed with length (with respect to $S$ ).

Let $V$ be an $n$-dimensional vector space over a field $\boldsymbol{F}$ of characteristic zero, and let $G$ be a subgroup of the general linear group $G L(V)$. Then $G$ acts naturally on the symmetric algebra $S\left(V^{*}\right)$, which may be identified with the polynomial ring $P_{n}=\boldsymbol{F}\left[x_{1}, \ldots, x_{n}\right]$. Let $\Lambda^{G}$ be the subalgebra of $G$-invariant polynomials, $I_{n}^{G}$ the ideal (of $P_{n}$ ) generated by the $G$ invariant polynomials without constant term, and $R^{G}:=P_{n} / I_{n}^{G}$ the associated coinvariant algebra. The coinvariant algebra is a direct sum of its homogeneous components, graded by degree: $R^{G}=\oplus_{k} R_{k}^{G}$. Let

$$
\operatorname{Hilb}_{G}(q):=\sum_{k \geq 0} \operatorname{dim} R_{k}^{G} q^{k}
$$

be the corresponding Hilbert series.

Definition 2.3. Let a be a (perfect) basis for a group $G \subset G L(V)$. Then $\mathbf{a}$ is a (perfect) Hilbertian basis for $G$ if

$$
\operatorname{Fmaj}_{(G, \mathbf{a})}(q)=\operatorname{Hilb}_{G}(q) .
$$

\subsection{Main Result}

Let $r$ be a positive integer and let $p$ be a divisor of $r$. The complex reflection group $G(r, p, n)$ is defined in [19] as the following subgroup of index $p$ of the wreath product $G(r, n)=\mathbf{Z}_{r} 2 S_{n}$ :

$$
G(r, p, n):=\left\{g=\left(\left(c_{1}, \ldots, c_{n}\right) ; \pi\right) \in G(r, n) \mid \sum_{i=1}^{n} c_{i} \equiv 0 \quad(\bmod p)\right\} .
$$

For more information on these groups the reader is referred to [13]. For the coinvariant algebra and flag major index on these groups see [4].

The main result of this paper states:

Theorem 2.4. Every complex reflection group $G(r, p, n)$ with parameters satisfying $\operatorname{gcd}(n, p, r / p)=1$ has a perfect Hilbertian basis.

See Theorem 3.3 and Corollary 4.1 below. The special case $p=1$ (wreath product) was established in $[21,2,3]$.

It follows that all classical Weyl groups have perfect Hilbertian-Mahonian bases (Corollaries 4.2 and 4.3 below) and that the alternating subgroup of a Weyl group of type $B$ has a Mahonian basis (Proposition 4.5 below). On the other hand, if $\operatorname{gcd}(n, p, r / p)>1$ then $G(r, p, n)$ does not necessarily have a Hilbertian basis; see Section 5 below. 


\section{A Perfect Basis for Complex Reflection Groups}

Let $\mathbf{u}=\left(u_{n-1}, \ldots, u_{0}\right)$ be the following sequence of $n$ elements in $G(r, p, n)$ :

$$
u_{i}:=\left(\bar{c}_{i} ; t_{i}\right) \quad(0 \leq i \leq n-1),
$$

where $t_{0} \in S_{n}$ is the identity permutation,

$$
\begin{gathered}
t_{i}:=s_{i} s_{i-1} \cdots s_{1}=(i+1, i, \ldots, 1) \in S_{n} \quad(1 \leq i \leq n-1), \\
\bar{c}_{i}:=(1,0, \ldots, 0, \alpha p-1) \in \mathbf{Z}_{r}^{n} \quad(0 \leq i \leq n-2),
\end{gathered}
$$

and

$$
\bar{c}_{n-1}:=(1,0, \ldots, 0, p-1) .
$$

The integer $0 \leq \alpha<r / p$ will be chosen later.

Remark 3.1. All the results below still hold if we define, more generally,

$$
\bar{c}_{n-1}:=(1,0, \ldots, 0, \beta p-1),
$$

where $\beta$ is any integer satisfying $\operatorname{gcd}(\beta, r / p)=1$.

Remark 3.2. If $r=p$ then one can also take $\bar{c}_{n-1}:=(0, \ldots, 0)$.

The main result of this section is the following.

Theorem 3.3. If $\operatorname{gcd}(n, p, r / p)=1$ then there exists $0 \leq \alpha<r / p$ such that $\mathbf{u}$ above is a perfect basis for $G(r, p, n)$.

The rest of this section is devoted to proving this result, using the Chinese Remainder Theorem and the Principle of Inclusion-Exclusion. For a discussion of the extent to which the condition $\operatorname{gcd}(n, p, r / p)=1$ can be relaxed, see Section 5 below.

Lemma 3.4. Let $H$ be the subgroup of $G(r, p, n)$ generated by the elements $\left\{u_{i} \mid 0 \leq i \leq\right.$ $n-2\}$. Then $H$ is isomorphic to $G(r, n-1)$.

Proof of Lemma 3.4. Define a map $\phi: H \rightarrow G(r, n-1)$ by erasing, from each $\pi=(\bar{c} ; t) \in H$, the last coordinate of $\bar{c}$. Let $\psi(\pi)$ be that coordinate, so that $\psi: H \rightarrow \mathbf{Z}_{r}$. Since every $\pi \in H$ satisfies $|\pi(n)|=n$, it follows that $\phi$ and $\psi$ are group homomorphisms. Moreover, for each $\pi=\left(\left(c_{1}, \ldots, c_{n-1}, c_{n}\right) ; t\right) \in H$ :

$$
c_{n}=(\alpha p-1) \sum_{i=1}^{n-1} c_{i}
$$

since this property holds for the generators, and is invariant under the group operation in $H$. It follows that

$$
c_{1}=\ldots=c_{n-1}=0 \Longrightarrow c_{n}=0 \text {, }
$$


namely: $\phi$ is injective. It is also surjective, since

$$
\left\{\left((1,0, \ldots, 0) ; t_{i}\right): 0 \leq i \leq n-2\right\}
$$

is a perfect basis for $G(r, n-1)$, by Proposition 2.1 above. Thus $\phi$ is a group isomorphism.

Consider now the sequence $\mathbf{u}=\left(u_{n-1}, \ldots, u_{0}\right)$ defined above. Clearly

$$
o\left(u_{i}\right)=(i+1) r \quad(0 \leq i \leq n-2)
$$

and

$$
o\left(u_{n-1}\right)=n r / p .
$$

(The latter equality holds also if we use the definitions in Remark 3.1 or 3.2.)

The product of all these orders is $n ! r^{n} / p=|G(r, p, n)|$. If we show that all the products

$$
u_{n-1}^{k_{n-1}} \cdots u_{0}^{k_{0}} \quad\left(0 \leq k_{i}<o\left(u_{i}\right)\right)
$$

are distinct, then it will follow that $\mathbf{u}$ is a perfect basis for $G(r, p, n)$.

Assume that

$$
u_{n-1}^{k_{n-1}^{\prime}} \cdots u_{0}^{k_{0}^{\prime}}=u_{n-1}^{k_{n-1}^{\prime \prime}} \cdots u_{0}^{k_{0}^{\prime \prime}} \quad\left(0 \leq k_{i}^{\prime}, k_{i}^{\prime \prime}<o\left(u_{i}\right)\right) .
$$

We want to show that $k_{i}^{\prime}=k_{i}^{\prime \prime}(\forall i)$. It suffices to show that $k_{n-1}^{\prime}=k_{n-1}^{\prime \prime}$, since then

$$
u_{n-2}^{k_{n-2}^{\prime}} \cdots u_{0}^{k_{0}^{\prime}}=u_{n-2}^{k_{n-2}^{\prime \prime}} \cdots u_{0}^{k_{0}^{\prime \prime}}
$$

and, by (the proof of) Lemma 3.4 and Proposition 2.1, this implies $k_{i}^{\prime}=k_{i}^{\prime \prime}(0 \leq i \leq n-2)$.

Indeed, by assumption

$$
u_{n-1}^{k_{n-1}^{\prime}-k_{n-1}^{\prime \prime}}=\left[u_{n-2}^{k_{n-2}^{\prime \prime}} \cdots u_{0}^{k_{0}^{\prime \prime}}\right]\left[u_{n-2}^{k_{n-2}^{\prime}} \cdots u_{0}^{k_{0}^{\prime}}\right]^{-1} \in H .
$$

Let $k:=k_{n-1}^{\prime}-k_{n-1}^{\prime \prime}$; working modulo $o\left(u_{n-1}\right)$, we can assume that $0 \leq k<n r / p$. $u_{n-1}^{k} \in H$ implies that $\left|u_{n-1}^{k}(n)\right|=n$ and therefore, by considering the $S_{n}$-component of $u_{n-1}, n \mid k$. Denote $\tilde{k}:=k / n$. Then

$$
u_{n-1}^{k}=u_{n-1}^{n \tilde{k}}=((\tilde{k} p, \ldots, \tilde{k} p) ; I d),
$$

where $I d \in S_{n}$ is the identity permutation and $0 \leq \tilde{k}<r / p$. (If we use the definition in Remark 3.1 then $\tilde{k} p$ should be replaced here by $\tilde{k} \beta p$. If we use the definition in Remark 3.2 then $o\left(u_{n-1}\right)=n$, and the proof ends here.)

On the other hand, we can present $u_{n-1}^{k} \in H$ in the form

$$
u_{n-1}^{k}=u_{n-2}^{k_{n-2}} \cdots u_{0}^{k_{0}} \quad\left(0 \leq k_{i}<o\left(u_{i}\right)\right) .
$$

The natural projection $T: H \rightarrow S_{n-1}$, defined by $T((\bar{c} ; t)):=t$, is a group homomorphism mapping the perfect basis $\left(u_{n-2}, \ldots, u_{0}\right)$ of $H$ onto the perfect basis $\left(t_{n-2}, \ldots, t_{0}\right)$ of 
$S_{n-1}$. Since $T\left(u_{n-1}^{k}\right)=I d$, it follows that $o\left(t_{i}\right)=i+1$ divides $k_{i}$; let $\tilde{k}_{i}:=k_{i} /(i+1)$ $(0 \leq i \leq n-2)$. Now

$$
u_{i}^{i+1}=\left(v_{i} ; I d\right) \quad(0 \leq i \leq n-2)
$$

where

$$
v_{i}:=(\underbrace{1, \ldots, 1}_{i+1}, 0, \ldots, 0,(\alpha p-1)(i+1)) \in \mathbf{Z}_{r}^{n} .
$$

Thus

$$
u_{n-1}^{k}=u_{n-2}^{(n-1) \tilde{k}_{n-2}} \cdots u_{1}^{2 \tilde{k}_{1}} u_{0}^{\tilde{k}_{0}}=\left(\sum_{i=0}^{n-2} \tilde{k}_{i} v_{i} ; I d\right)
$$

So far we have

$$
\sum_{i=0}^{n-2} \tilde{k}_{i} v_{i}=(\tilde{k} p, \ldots, \tilde{k} p) \in \mathbf{Z}_{r}^{n} \quad\left(0 \leq \tilde{k}_{i}<\frac{o\left(u_{i}\right)}{i+1}=r, 0 \leq i \leq n-2\right) .
$$

Since $v_{0}, \ldots, v_{n-2} \in \mathbf{Z}_{r}^{n}$ are linearly independent, we conclude that

$$
\tilde{k}_{n-2}=\tilde{k} p
$$

while

$$
\tilde{k}_{i}=0 \quad(0 \leq i \leq n-3)
$$

Thus

$$
\tilde{k} p v_{n-2}=(\tilde{k} p, \ldots, \tilde{k} p)
$$

Comparing the last coordinate on each side, we get by (4):

$$
\tilde{k} p(\alpha p-1)(n-1)=\tilde{k} p \quad\left(\text { in } \mathbf{Z}_{r}\right) .
$$

(Multiply both sides by $\beta$ for Remark 3.1.) Rewriting $(\alpha p-1)(n-1)-1=(n-1) \alpha p-n$, this is equivalent to

$$
\tilde{k}[(n-1) \alpha p-n]=0 \quad\left(\text { in } \mathbf{Z}_{r / p}\right),
$$

where $0 \leq \tilde{k}<r / p$ and $0 \leq \alpha<r / p$. (Same equation for Remark 3.1, $\operatorname{since} \operatorname{gcd}(\beta, r / p)=$ 1.)

We want to show that there exists $0 \leq \alpha<r / p$ such that (5) necessarily implies $\tilde{k}=0$. Equivalently, we must find $\alpha$ such that

$$
\operatorname{gcd}(r / p,(n-1) \alpha p-n)=1
$$

If $r / p=1$, every $\alpha$ will do. In general, we want to show that the following "False Assumption" leads to a contradiction.

False Assumption: For every $0 \leq \alpha<r / p$,

$$
\operatorname{gcd}(r / p,(n-1) \alpha p-n)>1 \text {. }
$$


Lemma 3.5. If $q>1$ is a common divisor of $r / p,(n-1) \alpha p-n$ and $(n-1) \alpha^{\prime} p-n$, where $\alpha \neq \alpha^{\prime}$ and $\operatorname{gcd}\left(\alpha^{\prime}-\alpha, q\right)=1$, then $q$ divides $\operatorname{gcd}(n, p, r / p)$.

Proof of Lemma 3.5. By assumption, $q$ divides $\left((n-1) \alpha^{\prime} p-n\right)-((n-1) \alpha p-$ $n)=(n-1)\left(\alpha^{\prime}-\alpha\right) p$. Since $\operatorname{gcd}\left(\alpha^{\prime}-\alpha, q\right)=1, q$ divides $(n-1) p$. Thus $q$ divides $\alpha(n-1) p-((n-1) \alpha p-n)=n$, so that $\operatorname{gcd}(q, n-1)=1$. Hence $q$ divides $p$ as well, completing the proof of the lemma.

By the "False Assumption" above there exists, for each $0 \leq \alpha<r / p$, a common (prime) divisor of $r / p$ and $(n-1) \alpha p-n$.

Lemma 3.6. Assume that $\operatorname{gcd}(n, p, r / p)=1$, and denote

$$
Q:=\{q \text { prime } \mid q \text { divides } r / p \text { and }(n-1) \alpha p-n \text { for some } 0 \leq \alpha<r / p\} \text {. }
$$

Then, for any number of distinct primes $q_{1}, \ldots, q_{t} \in Q$, the number of integers $0 \leq \alpha<$ $r / p$ such that $(n-1) \alpha p-n$ is divisible by all of $q_{1}, \ldots, q_{t}$ is $r /\left(p q_{1} \cdots q_{t}\right)$.

Proof of Lemma 3.6. Let $q \in Q$, and assume that it divides $(n-1) \alpha p-n$. If $\alpha^{\prime}-\alpha$ is divisible by $q$, then clearly $q$ divides also $(n-1) \alpha^{\prime} p-n$. Conversely, if $\alpha^{\prime}-\alpha$ is not divisible by the prime $q$ then $\operatorname{gcd}\left(\alpha^{\prime}-\alpha, q\right)=1$. By Lemma 3.5, and since $\operatorname{gcd}(n, p, r / p)=1$ by assumption, $q$ does not divide $(n-1) \alpha^{\prime} p-n$. It follows that the number of $0 \leq \alpha<r / p$ divisible by any $q \in Q$ is exactly $r /(p q)$.

We now consider any number of distinct primes $q_{1}, \ldots, q_{t} \in Q$. Suppose that $q_{i}$ divides $(n-1) \alpha_{i} p-n(1 \leq i \leq t)$. By the above argument, an integer $\alpha$ has the property that $(n-1) \alpha p-n$ is divisible by all of the $q_{i}$ if and only if $\alpha$ solves the $t$ simultaneous modular equations

$$
\alpha \equiv \alpha_{i} \quad\left(\bmod q_{i}\right) \quad(1 \leq i \leq t)
$$

A solution exists, and is unique $\left(\bmod q_{1} \cdots q_{t}\right)$, by the Chinese Remainder Theorem. It follows that the number of $0 \leq \alpha<r / p$ divisible by all of $q_{1}, \ldots, q_{t}$ is exactly $r /\left(p q_{1} \cdots q_{t}\right)$.

We shall now wrap up, by counting the integers $0 \leq \alpha<r / p$ according to which primes $q \in Q$ divide $(n-1) \alpha p-n$. According to the "False Assumption", each $\alpha$ has at least one such q. By Lemma 3.6 and the Principle of Inclusion-Exclusion, counting gives

$$
\frac{r}{p}=\sum_{q \in Q} \frac{r}{p q}-\sum_{q_{1}<q_{2}} \frac{r}{p q_{1} q_{2}}+\sum_{q_{1}<q_{2}<q_{3}} \frac{r}{p q_{1} q_{2} q_{3}}-\ldots
$$

Rearrangement gives

$$
\frac{r}{p} \cdot \prod_{q \in Q}\left(1-\frac{1}{q}\right)=0
$$

which is clearly a contradiction, since $Q$ is a finite set of integers greater than 1 . This completes the proof of Theorem 3.3. 


\section{Identities}

\subsection{A Flag Major Index for $G(r, p, n)$}

$G(r, p, n)$ is a subgroup of $G(r, n)=\mathbf{Z}_{r} 2 S_{n}$, and thus acts naturally on the polynomial ring $P_{n}=\mathrm{C}\left[x_{1}, \ldots, x_{n}\right]$; here $S_{n}$ acts by permuting the variables $x_{1}, \ldots, x_{n}$, while each copy of $\mathbf{Z}_{r}$ acts by multiplying a suitable $x_{i}$ by a complex $r$-th root of unity. Denote the ring of $G(r, p, n)$-invariant polynomials in $P_{n}$ by $\Lambda_{r, p, n}$. Let $I_{r, p, n}$ be the ideal of $P_{n}$ generated by the elements of $\Lambda_{r, p, n}$ without constant term. The quotient $R_{r, p, n}:=P_{n} / I_{r, p, n}$ is the coinvariant algebra of $G(r, p, n)$. Each complex reflection group $G(r, p, n)$ acts naturally on its coinvariant algebra. Let $R_{r, p, n}^{(k)}$ be the $k$-th homogeneous component of the coinvariant algebra, $R_{r, p, n}=\oplus_{k} R_{r, p, n}^{(k)}$, and let

$$
\operatorname{Hilb}_{r, p, n}(q):=\sum_{k \geq 0} \operatorname{dim} R_{r, p, n}^{(k)} q^{k}
$$

be the corresponding Hilbert series. $\operatorname{Hilb}_{r, p, n}(q)$ was expressed in [4] as a generating function for $\mathrm{fmaj}_{G(r, n)}$ on a certain subset of the wreath product $G(r, n)$. Using Theorem 3.3 it will be shown that $\operatorname{Hilb}_{r, p, n}(q)$ may be expressed as a generating function for a natural flag major index on the group $G(r, p, n)$ itself. This generalizes results for $G(r, 1, n)$ which were proved in $[21,2,3]$.

Let $G:=G(r, p, n)$ with $\operatorname{gcd}(n, p, r / p)=1$. Recall the perfect basis $\mathbf{u}$ for $G$ from Theorem 3.3 and the flag major index fmaj $(G, \mathbf{u})$ from Definition (2).

Corollary 4.1. If $\operatorname{gcd}(n, p, r / p)=1$ then $\mathbf{u}$ is a perfect Hilbertian basis for $G(r, p, n)$; namely,

$$
\operatorname{Hilb}_{r, p, n}(q)=\operatorname{Fmaj}_{(G(r, p, n), \mathbf{u})}(q),
$$

where $\operatorname{Fmaj}_{(G(r, p, n), \mathbf{u})}(q):=\sum_{\pi \in S_{n}} q^{\operatorname{fmaj}_{(G(r, p, n), \mathbf{u})}(\pi)}$

Proof. By Theorem 3.3 and identity (3),

$$
\sum_{\pi \in S_{n}} q^{\mathrm{fmaj}_{G(r, p, n)}(\pi)}=[r]_{q}[2 r]_{q} \cdots[(n-1) r]_{q}[n r / p]_{q}
$$

where $[m]_{q}:=\frac{q^{m}-1}{q-1}$. On the other hand, it is known (see, e.g., [4]) that

$$
\operatorname{Hilb}_{r, p, n}(q)=[r]_{q}[2 r]_{q} \cdots[(n-1) r]_{q}[n r / p]_{q},
$$

completing the proof.

\subsection{Classical Weyl Groups}

Recall the three infinite series of classical Weyl group: the symmetric groups $S_{n}$ (Weyl groups of type $A$ ), the signed permutation groups (sometimes called hyperoctahedral 
groups) $B_{n}$ (Weyl groups of type $B$ ), and the even signed permutation groups $D_{n}$ (Weyl groups of type $D$ ). We shall use here square brackets for the one-line notation of permutations, namely write

$$
\pi=[\pi(1), \ldots, \pi(n)]
$$

for $\pi$ in $S_{n}, B_{n}$ or $D_{n}$; round parentheses will be used for the cycle notation.

\section{Corollary 4.2.}

1. Let

$$
\alpha_{i}:=(i, i-1, \ldots, 1)=[i, 1,2, \ldots, i-1, i+1, i+2, \ldots, n] \quad(2 \leq i \leq n)
$$

be permutations in $S_{n}$. Then $\mathbf{a}=\left(\alpha_{n}, \alpha_{n-1}, \ldots, \alpha_{2}\right)$ is a perfect Hilbertian basis for the symmetric group $S_{n}$.

2. Let

$$
\beta_{i}:=[-i, 1,2, \ldots, i-1, i+1, i+2, \ldots, n] \quad(1 \leq i \leq n)
$$

be signed permutations in $B_{n}$. Then $\mathbf{b}=\left(\beta_{n}, \beta_{n-1}, \ldots, \beta_{1}\right)$ is a perfect Hilbertian basis for the hyperoctahedral group $B_{n}$.

3. Let

$$
\delta_{i}:=[-i, 1,2, \ldots, i-1, i+1, i+2, \ldots,-n] \quad(1 \leq i \leq n-1)
$$

and

$$
\delta_{n}:=[n, 1,2, \ldots, n-1]
$$

be signed permutations in $D_{n}$. Then $\mathbf{d}=\left(\delta_{n}, \delta_{n-1}, \ldots, \delta_{1}\right)$ is a perfect Hilbertian basis for the group of even signed permutations $D_{n}$.

Proof. By Theorem 3.3 and Remark 3.2, a, b and $\mathbf{d}$ are perfect bases for $S_{n}=G(1,1, n)$, $B_{n}=G(2,1, n)$ and $D_{n}=G(2,2, n)$, respectively (using $\alpha=1$ for $B_{n}$ and $D_{n}$, with Remark 3.2 for $\left.D_{n}\right)$. By Corollary 4.1, these bases are Hilbertian.

Corollary 4.3. $\quad$ 1. The sequence $\mathbf{b}$ is a perfect Mahonian basis for $B_{n}$ (with respect to the Coxeter generating set $S)$. Namely, the resulting flag major index fmaj $_{\left(B_{n}, \mathbf{b}\right)}$ is equidistributed with the length function $\ell_{\left(B_{n}, S\right)}$ over $D_{n}$.

2. The sequence $\mathbf{d}$ is a perfect Mahonian basis for $D_{n}$ (with respect to the Coxeter generating set $\left.S^{\prime}\right)$. Namely, the resulting flag major index fmaj ${ }_{\left(D_{n}, \mathbf{d}\right)}$ is equidistributed with the length function $\ell_{\left(D_{n}, S^{\prime}\right)}$ over $D_{n}$.

Proof. It is well known that for every Weyl group $W$, the Hilbert series of the coinvariant algebra of $W$ is equal to the Poincaré series of $W$, namely to the generating function for length with respect to the Coxeter generators; see, e.g., [14, §3.15]. Combining this with Corollary 4.1 gives the desired result.

While the statements on types $A$ and $B$ are not new, see [3], the statements on type $D$ (Corollary 4.2(3) and Corollary 4.3(2)) are new. In particular, note that $\mathrm{fmaj}_{\left(D_{n}, \mathbf{u}\right)}$ is equidistributed with, but different from, the flag major index for $D_{n}$ which was introduced by Biagioli and Caselli [5]. 


\subsection{The Alternating Subgroup of $B_{n}$}

Let $B_{n}^{+}$be the alternating subgroup of the Coxeter group of type $B$; namely, the subgroup consisting all elements in $B_{n}$ of even length.

Let $r_{1}:=[2,-1,3, \ldots, n]$ and $r_{i}:=[-1,2, \ldots, i+1, i, i+2, i+3, \ldots, n](2 \leq i \leq n-1)$. $R:=\left\{r_{i} \mid 1 \leq i \leq n-1\right\}$ is a set of generators for $B_{n}^{+}$with Coxeter-like relations [9, Chapter IV Section 1 Exercise 9]. The defining relations are:

$$
\begin{gathered}
r_{1}^{4}=1 \\
r_{i}^{2}=1 \quad(1<i<n) \\
\left(r_{i} r_{i+1}\right)^{3}=1 \quad(1 \leq i<n) . \\
\left(r_{i} r_{j}\right)^{2}=1 \quad(|i-j|>1)
\end{gathered}
$$

Let $\ell_{\left(B_{n}^{+}, R \cup R^{-1}\right)}(\pi)$ be the length of $\pi \in B_{n}^{+}$with respect to $R \cup R^{-1}$. Let

$$
v_{n}:=((0, \ldots, 0,1) ; I d)=[1,2, \ldots,-n] \in B_{n},
$$

and define a map $\psi: D_{n} \mapsto B_{n}^{+}$by

$$
\psi(w):= \begin{cases}w & \text { if } w \in B_{n}^{+} \\ w v_{n} & \text { if } w \notin B_{n}^{+} .\end{cases}
$$

Namely, $\psi$ switches the sign of the last letter of $w$ if $w \notin B_{n}^{+}$.

Fact 4.4. $\psi$ is a bijection.

Recall the basis $\mathbf{d}=\left(\delta_{n}, \ldots, \delta_{1}\right)$ for $D_{n}$ from Corollary $4.2(3)$ and let

$$
\gamma_{i}:=\psi\left(\delta_{i}\right) \quad(1 \leq i \leq n)
$$

Proposition 4.5. (1). The sequence $\mathbf{c}=\left(\gamma_{1}, \ldots, \gamma_{n}\right)$ is a Mahonian basis for $B_{n}^{+}$. Namely

(i) Every element $\pi \in B_{n}^{+}$has a unique presentation

$$
\pi=\gamma_{n}^{k_{n}} \gamma_{n-1}^{k_{n-1}} \cdots \gamma_{1}^{k_{1}} \quad 0 \leq k_{i} \leq 2 i \text { for } 1 \leq i<n \text { and } 0 \leq k_{n}<n \text {. }
$$

(ii)

$$
\sum_{\pi \in B_{n}^{+}} q^{\text {fmaj }_{\left(B_{n}^{+}, \mathbf{c}\right)}^{(\pi)}}=\sum_{\pi \in B_{n}^{+}} q^{\ell\left(B_{n}^{+}, R \cup R^{-1}\right)}(\pi) .
$$

(2). The flag major index is invariant under $\psi$. Namely, for every $w \in D_{n}$

$$
\operatorname{fmaj}_{\left(D_{n}, \mathbf{d}\right)}(w)=\operatorname{fmaj}_{\left(B_{n}^{+}, \mathbf{c}\right)}(\psi(w)) .
$$

(3). For every $w \in D_{n}, \operatorname{fmaj}_{\left(D_{n}, \mathbf{d}\right)}(w) \equiv 0(\bmod 2)$ if and only if $w \in D_{n} \cap B_{n}^{+}$. Similarly, for every $w \in B_{n}^{+}$, fmaj $\left.\operatorname{fB}_{n}^{+}, \mathbf{c}\right)(w) \equiv 0(\bmod 2)$ if and only if $w \in D_{n} \cap B_{n}^{+}$. 
Proof. Let $w$ be an element in $D_{n}$. By Corollary $4.2(3)$, there exist unique $0 \leq k_{i}<2 i$ $(0 \leq i<n)$ and $0 \leq k_{n}<n$ such that $w=\delta_{n}^{k_{n}} \cdots \delta_{1}^{k_{1}}$. Noticing that $v_{n}$ commutes with $\delta_{i}$ for $i<n$ we obtain

$$
\begin{gathered}
\gamma_{n}^{k_{n}} \cdots \gamma_{1}^{k_{1}}=\left(\delta_{n} v_{n}\right)^{k_{n}} \cdots\left(\delta_{1} v_{n}\right)^{k_{1}}=\delta_{n}^{k_{n}} \cdots \delta_{1}^{k_{1}} v_{n}^{\sum_{i} k_{i}} \\
=w v_{n}^{\mathrm{fmaj}_{\left(D_{n}, \mathbf{d}\right)}(w)}=w v_{n}^{\mathrm{fmaj}_{\left(D_{n}, \mathbf{d}\right)}(w) \bmod 2} .
\end{gathered}
$$

But $\gamma_{n}^{k_{n}} \cdots \gamma_{1}^{k_{1}} \in B_{n}^{+}$while $v_{n} \notin B_{n}^{+}$.

It follows that $w \in B_{n}^{+}$if and only if $\operatorname{fmaj}_{\left(D_{n}, \mathbf{d}\right)}(w) \bmod 2=0$. Hence

$$
\gamma_{n}^{k_{n}} \cdots \gamma_{1}^{k_{1}}=w v_{n}^{\mathrm{fmaj}_{\left(D_{n}, \mathbf{d}\right)}(w) \bmod 2}=\psi(w) .
$$

Since $\psi$ is a bijection this proves $(i),(2)$ and $(3)$.

To prove $(i i)$ recall from $[10]$ the bijection $\theta: B_{n}^{+} \mapsto D_{n}$

$$
\theta(w):= \begin{cases}w & \text { if } w \in B_{n}^{+} \\ w s_{0} & \text { if } w \notin B_{n}^{+},\end{cases}
$$

which switches the sign of the first letter of $w$ if $w \notin D_{n}$. By [10, Corollary 5.2(i)], the length is invariant under $\theta$. Namely, for every $w \in B_{n}^{+}$

$$
\ell_{\left(B_{n}^{+}, R \cup R^{-1}\right)}(w)=\ell_{\left(D_{n}, S^{\prime}\right)}(\theta(w)) .
$$

Combining (9), (10) with Corollary 4.3(2) and the fact that $\psi$ and $\theta$ are bijections we obtain

$$
\begin{aligned}
& \sum_{\pi \in B_{n}^{+}} q^{\mathrm{fmaj}_{\left(B_{n}^{+}, \mathbf{c}\right)}(\pi)}=\sum_{\pi \in B_{n}^{+}} q^{\mathrm{fmaj}_{\left(D_{n}, \mathbf{d}\right)}\left(\psi^{-1}(\pi)\right)}=\sum_{w \in D_{n}} q^{\mathrm{fmaj}_{\left(D_{n}, \mathbf{d}\right)}(w)}= \\
& =\sum_{w \in D_{n}} q^{\ell_{\left(D_{n}, S^{\prime}\right)}(w)}=\sum_{w \in D_{n}} q^{\ell_{\left(B_{n}^{+}, R \cup R^{-1}\right)}\left(\theta^{-1}(w)\right)}=\sum_{\pi \in B_{n}^{+}} q^{{ }_{\left(B_{n}^{+}, R \cup R^{-1}\right)}(\pi)} .
\end{aligned}
$$

This completes the proof of $(i i)$.

Remarks. 1. $\left(\gamma_{n}, \ldots, \gamma_{1}\right)$ is a perfect Mahonian basis for $B_{n}^{+}$if and only if $n$ is odd. If $n$ is even then the order of $\gamma_{n}$ is $2 n$ while $k_{n}$ is bounded by $n$ in (7), so $B_{n}^{+}$is not decomposed into a set-wise direct product of the cyclic subgroups generated by $\gamma_{n}, \ldots, \gamma_{1}$; in this case $\left(\gamma_{n}, \ldots, \gamma_{1}\right)$ is a Mahonian basis for $B_{n}^{+}$which is not perfect.

2. A major index and a Mahonian identity on the alternating subgroup of $S_{n}$ may be found in [17]. It should be noted that, while the length function is defined there with respect to a generating set analogous to the above $R \cup R^{-1}$, there is apparently no simple interpretation, involving bases, of the major index in this case. 


\section{Complex Reflection Groups with No Hilbertian Basis}

Proposition 5.1. For any prime $p$, the group $G\left(p^{2}, p, p\right)$ has no perfect Hilbertian basis.

Proof. Assume that $p$ is a prime number for which $G\left(p^{2}, p, p\right)$ has a perfect Hilbertian basis. A Hilbert function of the form (6) has a unique decomposition into factors of the form $\left[m_{i}\right]_{q}$, where $m_{i}$ are positive integers. It follows that, up to reordering, the $p$ elements $t_{0}, t_{1}, \ldots, t_{p-1}$ in a perfect Hilbertian basis for $G\left(p^{2}, p, p\right)$ have orders $o\left(t_{0}\right)=p^{2}$, $o\left(t_{1}\right)=2 p^{2}, \ldots, o\left(t_{p-2}\right)=(p-1) p^{2}$ and $o\left(t_{p-1}\right)=p^{2}$. Let $t_{i}=\left(v_{i} ; \pi_{i}\right)$, where $v_{i} \in\left(\mathbf{Z}_{p^{2}}\right)^{p}$ with sum of entries $\equiv 0(\bmod p)$ and $\pi_{i} \in S_{p}(0 \leq i \leq p-1)$.

Both $t_{0}$ and $t_{p-1}$ are of order $p^{2}$, and therefore neither $\pi_{0}$ nor $\pi_{p-1}$ contains a cycle of any size $1<i<p$. Each of them is, therefore, either a $p$-cycle or the identity permutation. If $\pi_{0}$ is a $p$-cycle then $t_{0}^{p}=\left(w_{0} ; I d\right)$ where $w_{0}=(\alpha, \ldots, \alpha), \alpha \equiv 0(\bmod p)$ but $\alpha \neq \equiv$ $\left(\bmod p^{2}\right)$. If $\pi_{0}=I d$ then $t_{0}^{p}=\left(w_{0} ; I d\right)$ where all the entries of $w_{0}$ are $0(\bmod p)$ but not all are $0\left(\bmod p^{2}\right)$, and their sum is $0\left(\bmod p^{2}\right)$. In both cases, $w_{0} \in\left(p \mathbf{Z}_{p^{2}}\right)^{p}$ is a nonzero vector whose sum of entries is $0\left(\bmod p^{2}\right)$. The same conclusion holds for $w_{p-1}$, where $t_{p-1}^{p}=\left(w_{p-1} ; I d\right)$

Now let $1<i<p$. Then $o\left(t_{i-1}\right)=i p^{2}$, and therefore $o\left(\pi_{i-1}\right) \mid i p^{2}$. If $\pi_{i-1}$ is a $p$-cycle then $t_{i-1}^{p^{3}}=I d$; and since $\operatorname{gcd}(i, p)=1$ this implies $t_{i-1}^{p^{2}}=I d$, contradicting $1<i<p$. Thus $\pi_{i-1}$ is not a $p$-cycle, and therefore $o\left(\pi_{i-1}\right) \mid i$. Denoting $t_{i-1}^{i p}=\left(w_{i-1} ; I d\right)(1<i<p)$, it follows that $w_{i-1} \in\left(p \mathbf{Z}_{p^{2}}\right)^{p}$ is a nonzero vector whose sum of entries is $0\left(\bmod p^{2}\right)$.

We conclude that all the vectors $w_{0}, w_{1}, \ldots, w_{p-2}, w_{p-1}$ belong to

$$
V:=\left\{w=\left(\alpha_{1}, \ldots, \alpha_{p}\right) \in\left(p \mathbf{Z}_{p^{2}}\right)^{p} \mid \alpha_{1}+\ldots+\alpha_{p}=0\right\}
$$

which is a $(p-1)$-dimensional vector space over the field $\mathbf{Z}_{p}$. The unique presentation property of the basis $t_{0}, \ldots, t_{p-1}$ implies that these $p$ vectors are linearly independent over $\mathbf{Z}_{p}$. This is a contradiction which completes the proof of the proposition.

\section{References}

[1] R. M. Adin, F. Brenti and Y. Roichman, Descent numbers and major indices for the hyperoctahedral group, Special issue in honor of Dominique Foata's 65th birthday (Philadelphia, PA 2000), Adv. Appl. Math. 27 (2001), 210-224.

[2] R. M. Adin and Y. Roichman, A flag major index for signed permutations, Proc. 11th Conference on Formal Power Series and Algebraic Combinatorics, Universitat Politècnica de Catalunya, Barcelona 1999, 10-17.

[3] R. M. Adin and Y. Roichman, The flag major index and group actions on polynomial rings, Europ. J. Combin. 22 (2001), 431-446.

[4] E. Bagno and R. Biagioli, Colored-descent representations for complex reflection groups, Isreal J. Math. 160 (2007), 317-347. 
[5] R. Biagioli and F. Caselli, Invariant algebras and major indices for classical Weyl groups, Proc. London Math. Soc. 88 (2004), 603-631.

[6] R. Biagioli and F. Caselli, A descent basis for the coinvariant algebra of type D, J. Algebra 275 (2004), 517-539.

[7] A. Björner and F. Brenti, Combinatorics of Coxeter Groups, Graduate Texts in Mathematics, Vol. 231, Springer-Verlag, 2005.

[8] A. Björner and M. L. Wachs, Permutation statistics and linear extensions of posets, J. Combin. Theory (Ser. A) 58 (1991), 85-114.

[9] N. Bourbaki, Lie Groups and Lie Algebras, English translation by Andrew Pressley, Springer, 2002.

[10] F. Brenti, V. Reiner and Y. Roichman, On the alternating subgroups of Coxeter groups, preprint, 2006.

[11] D. Foata and M. P. Schützenberger, Major index and inversion number of permutations, Math. Nachr. 83 (1978), 143-159.

[12] A. M. Garsia and I. Gessel, Permutation statistics and partitions, Adv. Math. 31 (1979), 288-305.

[13] M. Geck and G. Malle, Reflection Groups, Handbook of Algebra, Vol. 4, NorthHolland, Amsterdam, to appear.

[14] J. E. Humphreys, Reflection Groups and Coxeter Groups, Cambridge Studies in Advanced Math., No. 29, Cambridge Univ. Press, Cambridge, 1990.

[15] P. A. MacMahon, Combinatory Analysis I-II, Cambridge Univ. Press, London/NewYork, 1916. (Reprinted by Chelsea, New-York, 1960.)

[16] A. Muranov, On torsion-free groups with finite regular file bases, preprint, 2006.

[17] A. Regev and Y. Roichman, Permutation statistics on the alternating group, Adv. Appl. Math. 33 (2004), 676-709.

[18] R. Shwartz, Ordered generating systems, preprint, 2006.

[19] G. C. Shephard and J. A. Todd, Finite unitary reflection groups, Canadian J. Math. 6 (1954), 274-304.

[20] E. Steingrímsson, Permutation statistics of indexed permutations, Europ. J. Combin. 15 (1994), 187-205.

[21] J. Stembridge, On the eigenvalues of representations of reflection groups and wreath products, Pacific J. Math. 140 (1989), 353-396. 